\title{
Georreferenciación de las termales con alternativas de desarrollo para el Turismo de Bienestar en la región Cundinamarca
}

\author{
Thermal Georeferencing with Development Alternative for \\ Wellness Tourism in Cundinamarca Region
}

\section{Saudy Giovanna Niño Bernal ${ }^{1}$ \\ Sonia Duarte Bajaña ${ }^{2}$}

Para citar este artículo, utilice el nombre completo así:

Niño, S. G. \& Duarte, S. (2015). Georreferenciación de las termales con alternativas de desarrollo para el Turismo de Bienestar en la región Cundinamarca. Perspectiva Geográfica, 20(1), 203224.

\section{Resumen}

Este artículo muestra el resultado de la georreferenciación de las termales con alternativas de desarrollo para Turismo de Bienestar, presentes en el departamento de Cundinamarca. Propone evidenciar y categorizar las potencialidades que tendrá la región, acorde con la diversidad hidrológica, natural y cultural que hacen, de este territorio, un lugar con amplias apuestas productivas. Además, se pretende visibilizar dichos recursos, al reconocer que Colombia busca posicionarse como un destino de talla mundial en Turismo de Bienestar. Es así como, a manera de producto, se genera un mapa que evidencia la ubicación de las termales con potencial turístico, resultado del

1 Administradora Turística y Hotelera de la Fundación Universitaria los Libertadores, Bogotá (Colombia), Joven Investigadora cofinanciada por Colciencias en el marco del proyecto de Investigación, denominado: Alternativas de Desarrollo de Turismo de Bienestar no Visibles en la Región Bogotá, Cundinamarca. sgninob@libertadores.edu.co

2 Magíster en Investigación en Problemas Sociales Contemporáneos de la Fundación Universidad Central y Trabajadora Social de la Fundación Universitaria Monserrate, Bogotá, Colombia, sduarteb@libertadores.edu.co 
análisis que permitió determinar las características y elementos considerados como alternativa de desarrollo para esta región.

Palabras clave: Cundinamarca, georreferenciación, termales, Turismo de Bienestar.

\begin{abstract}
This article shows the result of thermal georeferencing with development alternatives for Wellness Tourism present in the department of Cundinamarca. It is proposed to highlight and categorize the potential of the region considering the hydrological, natural and cultural diversity that make this territory a place with ample productive opportunities. It is further intended make those resources visible, recognizing that Colombia seeks to position itself as a worldclass destination for Wellness Tourism. Therefore, as a type of product a map is generated showing the location of thermal tourism potential, the results of the analysis identifies the features and elements that were considered as an alternative development for the region.
\end{abstract}

Keywords: Cundinamarca, georeferencing, thermal, Wellness Tourism. 


\section{Introducción}

La oferta turística, entendida como el conjunto de bienes y servicios ofrecidos al turista en el departamento de Cundinamarca, es amplia; esta oferta se encuentra compuesta por la diversidad paisajística, la flora, la fauna, la variedad cultural, hidrológica y gastronómica, $\mathrm{y}$ hace de este territorio un lugar rico en apuestas productivas; una de ellas es el Turismo de Bienestar, entendido como los viajes encaminados al cuidado y al descanso personal; esta tipología turística se ha convertido en una de las estrategias con las que cuenta el país para posicionarse como un destino turístico reconocido en el ámbito nacional y en el internacional. La presente investigación muestra la georreferenciación de uno de los aspectos clave de dicho turismo: el termalismo con alternativas de desarrollo.

Por lo anterior, el objetivo de esta investigación se fundamenta en categorizar las termales en la región de Cundinamarca respecto de sus posibilidades de desarrollo para el Turismo de Bienestar; se tiene en cuenta que el país presenta un Plan de Negocios para esta tipología turística, que no contempla al departamento como un territorio en el que se podría implementar proyectos prioritarios conducentes a conocer la práctica de esta nueva tendencia.

Por tanto, se plantea indagar, evidenciar y contrastar información referente a las termales de la región de Cundinamarca, que pueden ser potencializadas al visibilizar elementos importantes alrededor del recurso, como uno de los productos importantes en la puesta de valor del país.

Es por lo anterior que esta investigación se desarrolla en cuatro apartados, a saber: en primer lugar, se propone contextualizar la importancia de la georreferenciación, como una herramienta tecnológica en turismo. En segundo lugar, se presenta una mirada hacia las generalidades del Turismo de Bienestar en Colombia, para pasar, en un tercer momento, al reconocimiento del termalismo presente en la región de Cundinamarca; finalmente, categorizar las termales con potencial de desarrollo en torno del Turismo de Bienestar, georreferenciándose en el mapa de la región. 


\section{Metodología}

La revisión documental que se llevó a cabo para recabar información en torno de las termales existentes en la región de Cundinamarca, consistió en la primera estrategia metodológica que permitiría dar alcance a los objetivos planteados; se recopiló, así, información de fuentes secundarias, tales como documentos, revistas y libros.

El trabajo etnográfico de observación, se convirtió en la segunda parte metodológica; permitió reconocer las fuentes termales existentes. El objetivo de la observación, consistió en documentar imágenes de los municipios visitados, con el fin de identificar las características del territorio; el eje eran las termales, para generar, así, un instrumento con el cual analizar las siguientes categorías: tipo de administración, es decir, si la termal pertenecía al sector público o privado; generación de empleo en la comunidad, accesibilidad, cercanía a la cabecera municipal, planta turística (transporte-alojamiento-alimentación-recreación-servicios de apoyo), productos locales del municipio y características de los recursos hídricos; elementos importantes para georreferenciar las termales con alternativas de desarrollo para Turismo de Bienestar en Cundinamarca.

\section{La georreferenciación como he- rramienta tecnológica en turis- mo}

La tendencia en la industria turística se ha dirigido hacia el uso de Sistemas de Información Geográfica (SIG), los cuales se han convertido en una herramienta para visualizar, de forma abstracta $y$ precisa, los hechos y fenómenos de la superficie terrestre, a partir de datos espacialmente referenciados (López, Larios \& Campillo, 2008).

Estos datos son recogidos por medio de técnicas, como la georreferenciación, que permite el posicionamiento de objetos espaciales en un sistema de coordenadas, para obtener información que es analizada y modelada en mapas cartográficos (Méndez, 2012; Ibáñez, 2012).

De esta forma, tanto los SIG como las herramientas y técnicas que los componen han sido implementados en la industria turística, para capturar, almacenar y representar datos; se genera, así, la elaboración de indicadores territoriales, los inventarios, la sectorización y 
la caracterización de espacios, y el diseño de mapas turísticos, entre otras actividades que surgen del uso de estos elementos (Farsari \& Prastacos, 2004; Junior \& Almeida, 2009).

En Colombia, es importante que el sector turístico diseñe estrategias que le permitan potenciar el territorio cumpliendo, de tal forma, con uno de los objetivos propuestos en el Plan Sectorial de Turismo 20142018 que le apuesta a la: “(...) diversificación y especialización de la oferta, a través del desarrollo de productos competitivos, que se integren a los mercados globales bajo la sombrilla de la innovación tecnológica y mediados por el aprovechamiento responsable de los recursos naturales y culturales" (DNP, 2014, p. 40).

Para dar cumplimiento a este objetivo se sugiere implementar la georreferenciación, puesto que esta herramienta permitirá reconocer esos destinos junto a sus potencialidades, para el desarrollo de productos innovadores y diferenciadores que se posicionarían nacional e internacionalmente.

Además de esto, el país cuenta con ventajas competitivas, ya que po- see una riqueza turística amplia; por tanto, el reconocimiento y ubicación de la oferta regional que se encuentra disponibles para los pobladores y turistas, permitirá generar apuestas productivas enmarcadas en sectores competitivos, como lo es actualmente el Turismo de Bienestar.

\section{Generalidades del Turismo de Bienestar en Colombia}

En Colombia, el Turismo de Bienestar ha sido abordado desde el Ministerio de Comercio Industria y Turismo (MinCIT), con el fin de revisar cuál era su situación y prospectiva en el país. Para ello, contrató en el año 2012 a la firma Tourism $\&$ Leisure, con el fin de elaborar un Plan de Negocios, específicamente de bienestar, presentado en abril de 2013. A continuación, se muestra un resumen de los principales resultados que fueron fundamentales para la presente investigación.

El Turismo de Bienestar es entendido como aquellos viajes con el objetivo primordial de realizar actividades encaminadas al cuidado, a sentirse bien al recibir tratamientos alternativos y al descanso (Tourism \& Leisure, 2013); se afirma que se ha convertido en una 
tendencia en Colombia, y que el país cuenta con una serie de recursos que pueden ser potencializados para el aprovechamiento de esta apuesta productiva.

De igual manera, se plantea que el Turismo de Bienestar es un complemento del Turismo de Salud; además este segmento tiene tres productos claves: la talasoterapia, entendida como el uso del agua de mar y los elementos marinos; los centros de bienestar o spas, que son espacios dedicados a la relajación y con una serie de servicios alrededor de esta práctica, y el termalismo, que se entiende como el uso de aguas barros y lodos, dirigido a ofrecer un servicio en torno de tratamientos curativos y preventivos.

En este Plan de Negocios se enfatiza que el país tiene un alto potencial por desarrollar, ya que posee 310 fuentes, de las cuales 30 tienen capacidad para convertirse en centros termales y 900 en centros de bienestar. Cabe aclarar que se está utilizando solo un $6 \%$ de su potencial, lo cual evidencia que el Turismo de Bienestar en Colombia es casi inexistente (Euromonitor Internacional, 2012).
Es por lo anterior que en este plan se sugiere estrategias de negocios, para desarrollar productos sobre el Turismo de Bienestar, como uno de los principales motores en el desarrollo social, ambiental y económico en los contextos regional y nacional.

\section{El termalismo en la región de Cundinamarca}

Retomando lo anterior, Cundinamarca no ha sido visible en la propuesta mencionada; se desconoce que se ha configurado en un destino turístico competitivo en lo nacional e internacional debido a su ubicación central en el país, su riqueza hidrográfica, natural, cultural y ancestral (Gobernación de Cundinamarca, 2012).

En este contexto, el turismo constituye una posibilidad potencial y dinámica de la economía regional, con grandes ventajas para el departamento, porque permite mostrar y desarrollar los recursos y atributos con los que cuenta (Fontur, 2012). Específicamente, en torno de las termales, el Instituto Interamericano de Cooperación para la Agricultura (ICCA), en el libro, intitulado: Prácticas agrosostenibles para el departamento de Cundinamarca, 
hace referencia a la presencia de 40 fuentes termales en este territorio (ICCA, 2001).

De igual forma, un estudio químico preliminar sobre Cundinamarca - llevado a cabo por el Instituto Colombiano de Geología y Minería (Ingeominas), en el año 2003- muestra 52 fuentes, lo que invita a la implementación de estos recursos en la industria mineral natural y balneoterapia (AlfaroValero, 2005).

El reconocimiento de las posibilidades termales de la región se convierte en el elemento generador de la pregunta de investigación, enfocada en la existencia de termales con alternativas de desarrollo en Turismo de Bienestar, presentes en la región de Cundinamarca, con el fin de visibilizar estas apuestas para el impulso de productos innovadores.

De acuerdo con Ocampo (2004) y Tello (2006), la localización geográfica, la variedad de recursos y la intervención del Estado se presentan como alternativas de desarrollo fundamentales para el crecimiento de una región; en este sentido, reconocer las termales junto con sus bondades terapéuticas y los recursos alrededor, dará paso a la creación e implementación de nuevos proyectos que fomenten el crecimiento regional en el marco del Turismo de Bienestar.

Lo anterior, no solo muestra la presencia e importancia de las fuentes termales en la región, sino que también abre un escenario de investigación que ha sido poco abordado y que cuenta con posibilidades de desarrollo.

\section{Resultados}

La revisión documental inicial, generó como resultado la identificación de 39 municipios con presencia de 52 fuentes termales; sobre estos, se planteó un reconocimiento del territorio desde las categorías propuestas en la Matriz Oportunidades del Turismo de Bienestar (MOTB). ${ }^{3}$ A continuación, en la

3 La elaboración de la Matriz Oportunidades del Turismo de Bienestar, MOTBes una propuesta realizada en el marco del proyecto de Investigación, denominado: Alternativas de Desarrollo de Turismo de Bienestar no visibles en la Región Bogotá, Cundinamarca. Esta permite calificar los recursos disponibles en los municipios como fuentes termales, variedad de flora y frutas, clima, asentamientos étnicos, atractivos turísticos, saberes propios de la comunidad local (factores endógenos); política pública, ubicación geográfica, inversión central, influencia de territorios cercanos (factores exógenos), variables necesarias para el desarrollo de Turismo de Bienestar. 
Tabla 1, se presenta la relación de dichos municipios.

Tabla 1. Municipios de Cundinamarca con registro de termales.

\begin{tabular}{|c|c|c|c|}
\hline & MUNICIPIO & & MUNICIPIO \\
\hline 1 & Agua de Dios & 22 & Machetá \\
\hline 2 & Anapoima & 23 & Manta \\
\hline 3 & Apulo & 24 & Medina \\
\hline 4 & Bogotá & 25 & Nemocón \\
\hline 5 & Caparrapí & 26 & Nilo \\
\hline 6 & Chipaque & 27 & Nimaima \\
\hline 7 & Choachí & 28 & Pandi \\
\hline 8 & Chocontá & 29 & Paratebueno \\
\hline 9 & Cota & 30 & Ricaurte \\
\hline 10 & Cucunubá & 31 & San Francisco \\
\hline 11 & El Rosal & 32 & Suesca \\
\hline 12 & Fómeque & 33 & Tabio \\
\hline 13 & Gachalá & 34 & Tenjo \\
\hline 14 & Gachetá & 35 & Tibitiritá \\
\hline 15 & Girardot & 36 & Tocaima \\
\hline 16 & Guasca & 37 & Útica \\
\hline 17 & Guataquí & 38 & Villeta \\
\hline 18 & Guatavita & 39 & Yacopí \\
\hline 19 & Junín & & \\
\hline 20 & La Calera & & \\
\hline 21 & La Mesa & & \\
\hline
\end{tabular}

Fuente: Elaboración propia.

El trabajo de campo, en el que se propuso georreferenciar las termales con alternativas de desarrollo para el Turismo de Bienestar, en la región de Cundinamarca, inicia con el hallazgo de 19 municipios con la presencia de 27 fuentes termales; 39 municipios con 52 termales, como aparecía en los documentos de In- geominas (Alfaro, 2005) y Prácticas Agrosostenibles de Cundinamarca (ICCA, 2001). Esta diferencia se debe a situaciones como: termales secas, información errada, fuentes no identificadas o pertenecientes a un predio privado.

A continuación, se muestra la Tabla 2, que detalla la situación de los 39 municipios iniciales.

Tabla 2. Hallazgos iniciales.

\begin{tabular}{|c|c|}
\hline Municipios con termales secas & 7 \\
\hline Municipios con información errada & 7 \\
\hline $\begin{array}{c}\text { Municipios con termales no } \\
\text { georreferenciadas }\end{array}$ & 6 \\
\hline Municipios con termales & 19 \\
\hline
\end{tabular}

Fuente: Elaboración propia.

A partir de lo anterior, de la información obtenida desde los pobladores de la región, las Alcaldías municipales, de las autoridades turísticas, y del trabajo de observación, se encuentra que las termales existentes en municipios como Apulo, Cota, Gachalá, Guatavita, La Mesa y Villeta, existieron hace algunos años, pero debido al calentamiento global y a la falta de mantenimiento, se fueron secando.

Es importante mencionar el caso de Útica, en donde Ingeominas referencia tres termales; no obstante, el 
recorrido evidencia una sola que ha rios desconocen la historia de este sido convertida en baño medicinal recurso; sin embargo, el registro de agua azufrada. Durante el reco- fotográfico que se encuentra en las rrido se encuentra que, en la Alcal- paredes de la entidad muestra que día Municipal, algunos funciona- sí existieron estas fuentes termales.

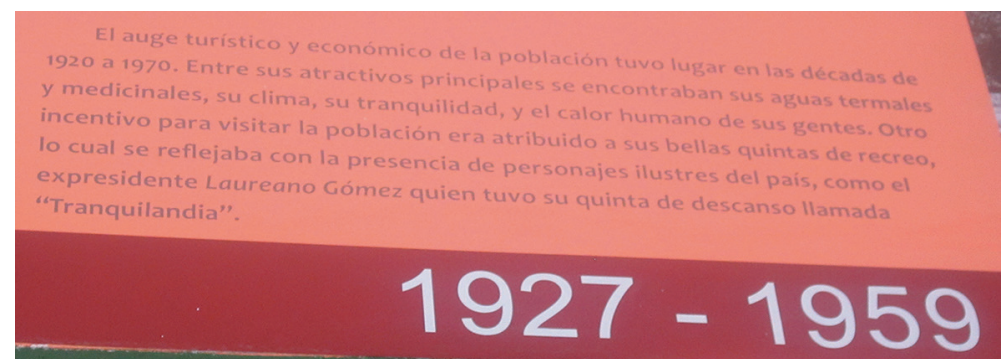

Figura 1. Registro fotográfico encontrado en la Alcaldía de Útica.

Fuente: Elaboración propia.

La Figura 1 hace parte de un registro histórico que explica la situación de Útica a través de los años, pero para la presente investigación es la evidencia que permite reconocer los acontecimientos en torno de la existencia de fuentes termales en este municipio; las catástrofes ambientales ocasionaron la desaparición de este recurso.

En segunda medida, se realiza una comparación entre el trabajo de campo y la información obtenida en los documentos; se evidencian termales que nunca existieron o pertenecen a otros territorios; en esta variable se encuentran municipios, como Cucunubá, Fómeque, Machetá, Manta, Medina, Nilo y Nimaima.
A manera de ejemplo, se encuentra la termal Los Volcanes, promocionada en el municipio de Machetá; realmente es de Chocontá.

En tercer lugar, no se georreferencian algunas de estas termales, puesto que presentan dificultades de acceso o pertenecen a una propiedad privada $^{4}$, cuyo dueño no tiene interés en desarrollar un producto turístico alrededor de dicho recurso; en esta variable se encuentran municipios como: La Calera, Bogotá, Guataquí, Yacopí y Tenjo.

$4 \quad$ Las termales que se encuentran para uso exclusivo de actores privados, no se tuvieron en cuenta dentro del estudio, ya que el interés de la investigación se enfoca en las alternativas de desarrollo que dichas termales generan para la región. 
Partiendo del reconocimiento de las termales en el territorio, y desde la matriz mencionada anteriormente, se procede a categorizarlas y a establecer las alternativas de desarrollo para el Turismo de Bienestar en la región de Cundinamarca; se analiza las siguientes variables: a) administración pública o privada, b) generación de empleo en la comunidad, c) accesibilidad, d) cercanía a la cabecera municipal, e) planta turística (transporte - alojamiento - alimentación - recreación - servicios de apoyo), f) productos locales del municipio y g) características de los recursos hídricos.

\section{a) Administración pública o privada}

Al realizar el reconocimiento de las fuentes termales en Cundinamarca, se evidencia que 12 son de administración privada; estas termales presentan una característica particular, puesto que la inversión en infraestructura ha sido desarrollada para el aprovechamiento del turismo, como una fuente de ingreso importante; en esta variable se encuentran los municipios de Agua de Dios, Anapoima, Choachí, Chocontá, Junín, Pandi, Ricaurte, Suesca, Tabio, Tibitiritá, Tocaima y Útica.

Por otro lado, se encuentra municipios en donde las termales tienen administración pública como $\mathrm{Ne}$ mocón; allí, se está desarrollando un proyecto financiado por el MinCIT a través de Fontur, que consiste en la construcción de un complejo termal para ampliar la oferta turística de esta región.

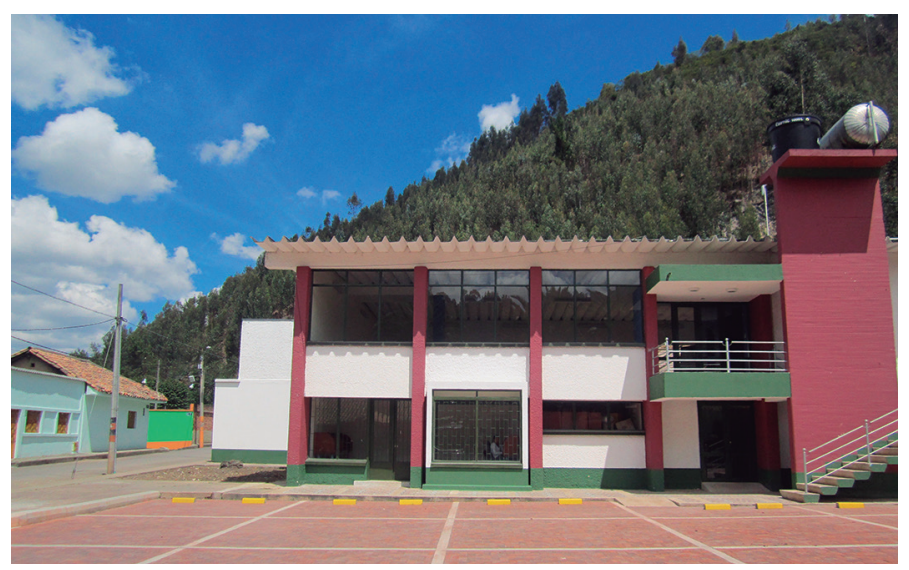

Figura 2. Fachada termal de Nemocón.

Fuente: Elaboración propia. 
También, existe termales que son administradas por una entidad pública, pero, a su vez, son subarrendadas. Es el caso de Guasca con la fuente termal Santa Bárbara, que está siendo remodelada para proponerse en un contrato de comodato; es decir, entregar este bien a un administrador particular que se encargue de promoverla para el sector turístico, y a partir de esto generar una contraprestación que debe ser la contratación de mano de obra del municipio.

Como último caso existen termales que hasta el momento no son administradas por una entidad pública o privada; es el caso del Rosal y Chipaque, estos municipios cuentan con un potencial importante para desarrollar productos enfocados hacia el termalismo, pero hace falta una administración que se haga cargo.

Es importante mencionar la termal la Rivera, perteneciente a los municipios de Junín y Gachetá. Este recurso hace algunos años tenía una administración privada; posteriormente, se reconoce como recurso público. Hasta el momento esta fuente se encuentra abandonada; se desaprovecha su potencial turístico; además, ya posee la infraestructura idónea -baños, piscina terminada, alojamiento y restaurante- para ser promocionada, tal como se muestra en las Figuras 3 y 4.

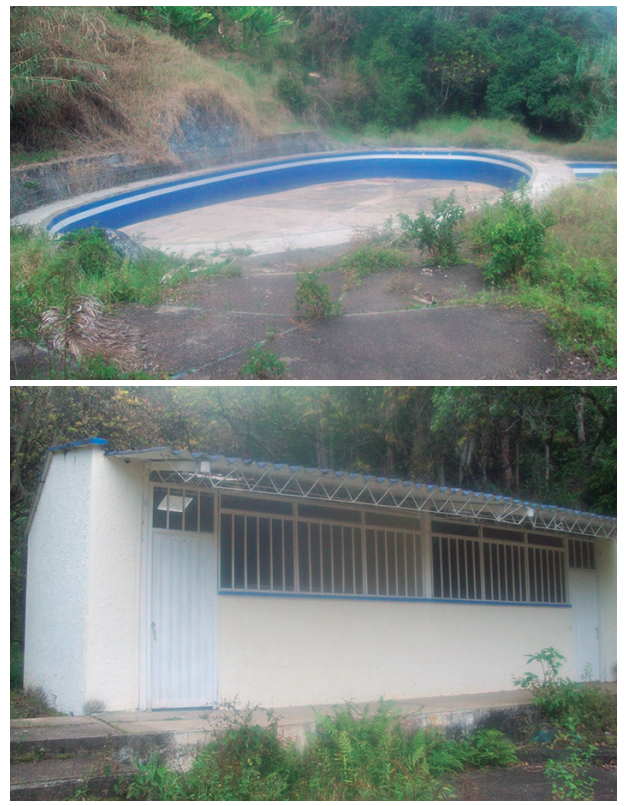

Figura 3. Termales la Rivera, JunínGachetá.

Fuente: Elaboración propia.

\section{b) Generación de empleo en la comunidad}

En esta categoría se presentan tres factores; en primer lugar, la contratación de mano de obra del municipio; en segunda medida, la contratación de pobladores de otros municipios, y por último negocios familiares en donde los empleados pertenecen al mismo núcleo familiar. 
Correspondiente al primer factor se encuentran municipios, como: Tocaima, Tabio, Choachí, Chocontá, Tibitiritá, Nemocón, Agua de Dios, Pandi, Paratebueno, Ricaurte y Guasca, en donde se contrata pobladores de los propios municipios para trabajar en las termales; se genera alrededor de 8 a 12 empleos directos. En este sentido, los trabajadores ocupan cargos operativos $\mathrm{y}$ administrativos, $\mathrm{y}$ garantizan el buen funcionamiento de la termal. Dentro del segundo factor, están las termales de Junín y Chocontá, en donde se evidencia la inexistencia de pobladores con conocimientos para ofrecer otros servicios o tratamientos enfocados hacia el bienestar; por tanto, para suplir esta necesidad deben recurrir a mano de obra de municipios aledaños.

Como último factor, al existir negocios familiares, la contratación de pobladores es mínima; este caso se presenta en municipios, como Anapoima, Útica y Suesca en donde el núcleo familiar trabaja alrededor de dicho recurso.

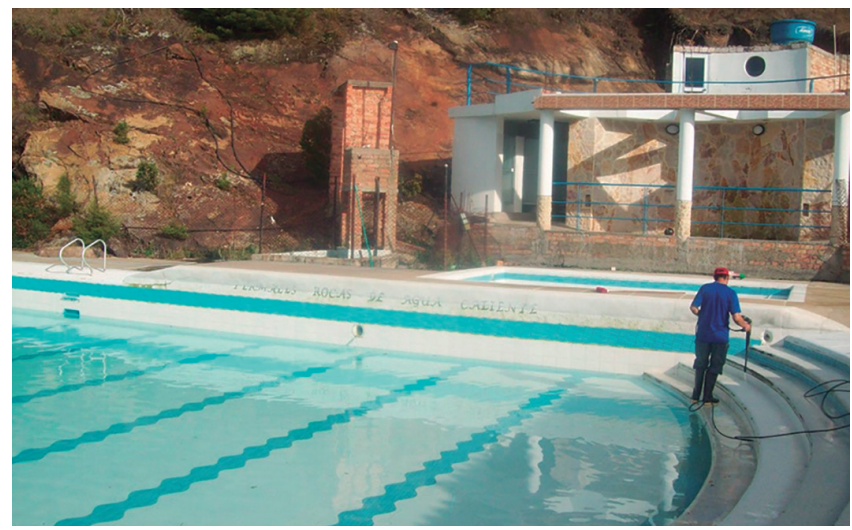

Figura 4. Trabajador limpia termal, Suesca.

Fuente: Elaboración propia.

\section{c) Accesibilidad}

En esta categoría se tiene en cuenta vías de acceso y señalización turística, como elementos fundamentales para promocionar un destino turístico; se evidencian dos situa- ciones: la primera tiene que ver con la presencia de vías de acceso pavimentadas y señalización turística adecuada; la segunda son las precarias condiciones de las vías de acceso y la carencia de señalización turística. 
En municipios, como Ricaurte, Tabio, Choachí, Suesca, Chipaque, Guasca, Chocontá, Tibitiritá, Anapoima, Nemocón, Junín, Gachetá, Útica, Paratebueno, Agua de Dios, Pandi y Tocaima, las vías de acceso están pavimentadas y poseen una adecuada señalización turística, lo cual facilita el acceso, reconocimiento y confiabilidad de las termales. Aunque en el área rural esta tendencia no está tan marcada, fue posible acceder a los lugares.

En segundo lugar, la falta de señalización turística es un factor determinante en los territorios. En este sentido, se encuentra el municipio de San Francisco; como se visualiza en la Figura 5, aparece en la carretera una señalización turística que indica la presencia de una fuente termal. Inicialmente, el aspecto de esta señal es descuidado; por otro lado, se presenta como la única indicación existente para llegar al destino; esto es insuficiente y ocasiona equivocaciones en la ruta.

La Figura 5 también muestra un acercamiento de la leyenda que aparece en esta señalización; allí se afirma que la termal se encuentra ubicada a una hora desde este punto; sin embargo, no existe ningún otro tipo de anuncio en el camino que indique la cercanía del lugar; de igual forma, la carretera no se encuentra en condiciones óptimas; por tanto, las expectativas al encontrar este tipo de señalización son amplias, pero el acceso al lugar no es posible debido a estas condiciones. Adicional a lo anterior, al llegar al punto indicado por la señalización se encuentra un camino destapado, sin ninguna información que indique la necesidad de realizar una caminata de, por lo menos, 10 minutos para llegar al destino.
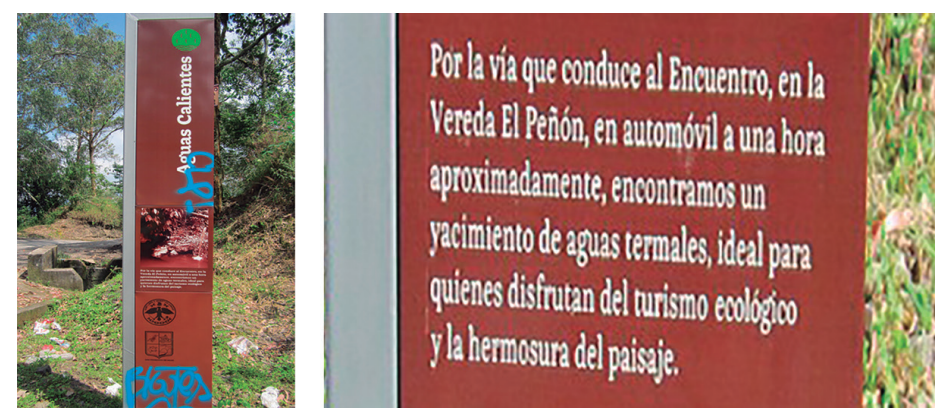

Figura 5. Señalización turística; se indica leyenda sobre la presencia de termales en el municipio de San Francisco.

Fuente: Elaboración propia. 
En municipios como El Rosal, Chipaque y Guataquíse encuentra falencias en cuanto la señalización $\mathrm{y}$ vías de acceso. En este último municipio, Ingeominas referencia unos pozos de lodo, a los cuales no fue posible acceder por tres razones: primera, este recurso no queda cerca de la cabecera municipal; segunda, la carretera no se encuentra en óptimas condiciones, y tercera, la señalización turística es precaria, lo cual impide la posibilidad de potencializar productos para el desarrollo del municipio.

\section{d) Cercanía a la cabecera muni- cipal}

En el trabajo de campo se encuentra que solo 7, de 27 termales, están ubicadas en la cabecera municipal, tal es el caso de Los Chorros en el municipio de Agua de Dios, Las Duchas de agua azufrada, presentes en el municipio de Útica, Baños medicinales de Santa Ana y Baños medicinales de Santa Lucía en Anapoima, el Complejo Termal de Nemocón, Termales el Zipa en Tabio, y Termales Santa Mónica en Choachí.

De igual forma, 20 termales se encuentran ubicadas en la parte rural de los municipios. Los pobladores no siempre las reconocen como propias; por el contrario, las asumen como pertenecientes al municipio aledaño, lo que ocasiona falta de apropiación y desaprovechamiento de su potencial. Esta es la situación del municipio de Ricaurte, en donde existen 2 complejos termales: Bosques de Babilonia y Casa Blanca, recursos que no se encuentran en la cabecera municipal. Aunque geográficamente le pertenecen a Ricaurte, la mayoría de pobladores asume que corresponden al municipio de Nilo.

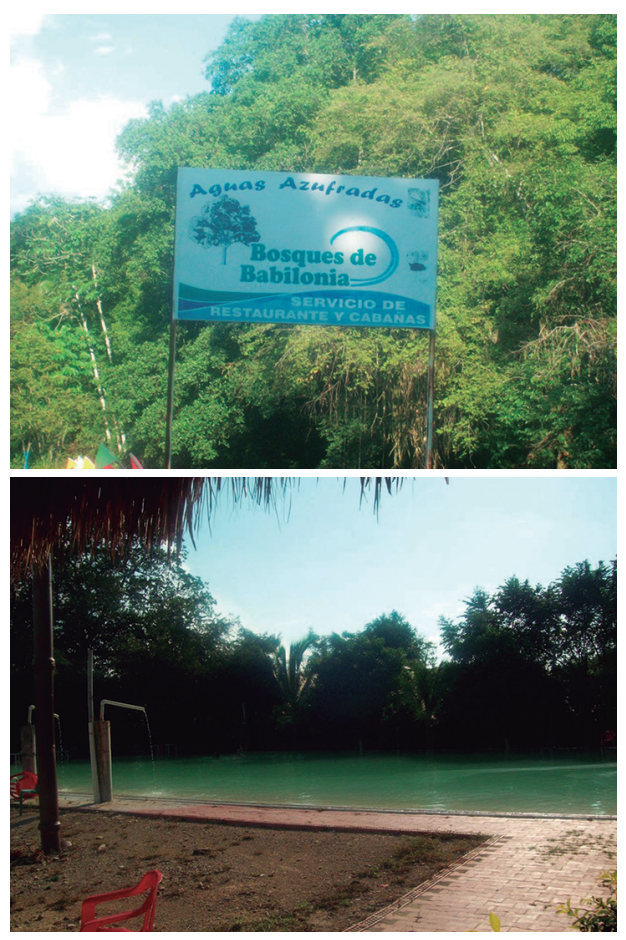

Figura 6. Bosques de Babilonia y Casablanca, Ricaurte.

Fuente: Elaboración propia. 
e) Planta turística (transporte alojamiento - alimentación - recreación - servicios de apoyo)

En municipios como Choachí, Tabio, Chocontá, Junín, Tibirita, Suesca, Tocaima, Ricaurte, Útica y Pandi se encuentra consolidada una planta turística alrededor de las termales; se ofrece a los visitantes un servicio completo para satisfacer sus necesidades. De esta forma se presta servicios de alojamiento, recreación, y alimentación alrededor de la termal.
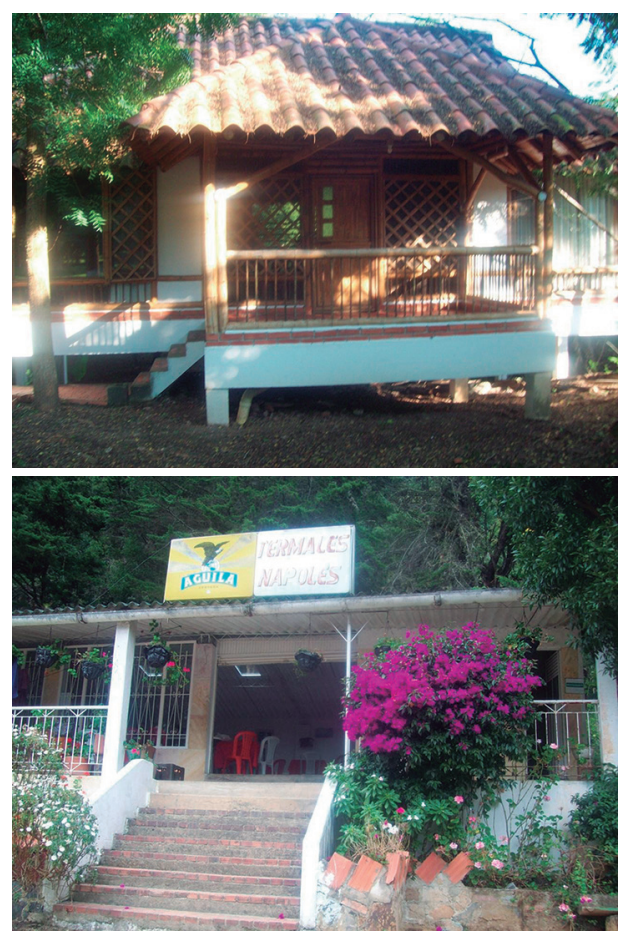

Figura 7. Bosques de Babilonia, Ricaurte, Restaurante Termales Nápoles, Chocontá. Fuente: Elaboración propia.
En municipios como Agua de Dios, Anapoima, Chipaque, El Rosal y San Francisco, no existe una planta turística consolidada, puesto que solamente se ofrece el servicio de la termal y no se desarrolla otro tipo de actividad alrededor de la misma.

\section{f) Productos locales del municipio}

El Turismo de Bienestar, en algunos destinos, ha involucrado el uso de productos locales; un ejemplo de esto se refleja en Asia, en donde se utilizan elementos naturales en diferentes tratamientos, como masajes, infusiones y baños para ofrecer otros servicios asociados con la termal (Laing \& Weiler, 2008).

En Cundinamarca existen municipios que son productores de hierbas aromáticas y frutas; Guasca es un municipio productor de fresas; San Francisco, de café y cítricos; Chipaque y Chocontá, de hierbas aromáticas; Tabio y Suesca, de flores.

Los productos locales, basados en experiencias como las de Asia, lograrían ser útiles y provechosos en la práctica de esta tipología turística en Cundinamarca, al involucrar estos elementos en turcos o cáma- 
ras de vapor, y en tratamientos de spa; se permitiría el desarrollo de otros sectores alrededor del termalismo y se generaría un ingreso adicional para la región.
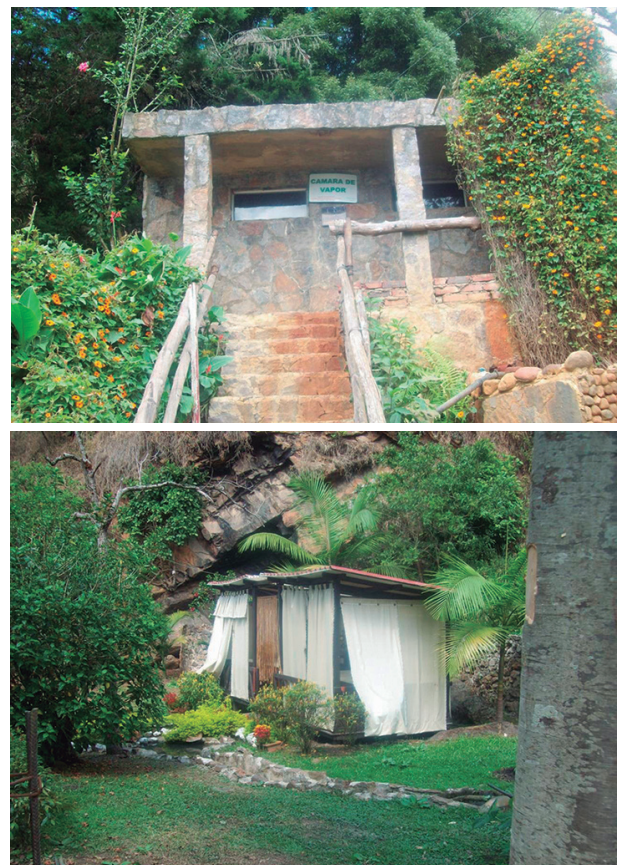

Figura 8. Cámara de vapor termal Nápoles, Chocontá; Spa Paraíso Termal, Tibirita

Fuente: Elaboración propia.

Las fotografías anteriores hacen parte de servicios ofrecidos alrededor de la termal, aunque no todos los lugares visitados ofrecen servicio de spa y turco; es la ejemplificación de los servicios en los que se puede involucrar productos locales para satisfacer otras necesidades de los turistas; de los lugares visitados se encuentra que en municipios como Chocontá, Tibirita, Suesca, Choachí y Tocaima se prestan estos servicios en torno de estas fuentes.

\section{g) Características de los recursos hídricos}

La presente investigación no tiene como fin efectuar un estudio químico sobre la composición de las aguas termales; no obstante, el trabajo de campo permitió conocer aspectos de estas que influyen en el tipo de turista que accede a las mismas; unas termales, con composición mineral sulfatada y reconocida, en parte, por su olor, y otras, con una composición mineral diferente, identificadas por la temperatura y color del agua.

A partir de lo anterior, se logra establecer que las termales con composición mineral sulfatada presentan el agua de color entre amarillo y rojo; como se mencionaba anteriormente, el olor es fuerte y penetrante. Las personas que acceden a este servicio son, por lo regular, mayores, quienes buscan sanar enfermedades relacionadas con el sistema nervioso y muscular, pues estas son las propiedades atribuidas a estas aguas. 
Por otro lado, las termales de diferente composición mineral, que aparecen con colores transparentes, amarillos y verdes, sin un olor distintivo, son el lugar de atracción de pobladores y turistas que buscan el bienestar.
A continuación, se muestra la relación de las termales en la región de Cundinamarca, con respecto a las características mencionadas.

Tabla 3. Clasificación de las termales en Cundinamarca.

\begin{tabular}{|c|c|c|c|}
\hline & MUNICIPIO & $\begin{array}{c}\text { TERMALES } \\
\text { CON OTRA } \\
\text { COMPOSICIÓN } \\
\text { MINERAL }\end{array}$ & $\begin{array}{c}\text { TERMALES CON } \\
\text { COMPOSICIÓN } \\
\text { MINERAL } \\
\text { SULFATADAS }\end{array}$ \\
\hline 1 & Agua de Dios & & $\mathrm{X}$ \\
\hline 2 & Anapoima & & $\mathrm{X}$ \\
\hline 3 & Chipaque & $\mathrm{X}$ & \\
\hline 4 & Choachí & $\mathrm{X}$ & \\
\hline 5 & Chocontá & $\mathrm{X}$ & \\
\hline 6 & El Rosal & $\mathrm{X}$ & \\
\hline 7 & Gachetá & $\mathrm{X}$ & \\
\hline 8 & Guasca & $\mathrm{X}$ & \\
\hline 9 & Junín & $\mathrm{X}$ & \\
\hline 10 & Nemocón & $\mathrm{X}$ & \\
\hline 11 & Pandi & $\mathrm{X}$ & $\mathrm{X}$ \\
\hline 12 & Paratebueno & $\mathrm{X}$ & \\
\hline 13 & Ricaurte & & $\mathrm{X}$ \\
\hline 14 & San Francisco & $\mathrm{X}$ & \\
\hline 15 & Suesca & $\mathrm{X}$ & \\
\hline 16 & Tabio & $\mathrm{X}$ & \\
\hline 17 & Tibitiritá & $\mathrm{X}$ & \\
\hline 18 & Tocaima & & $\mathrm{X}$ \\
\hline 19 & Útica & & $\mathrm{X}$ \\
\hline
\end{tabular}

Fuente: Elaboración propia. 
En la tabla anterior se muestra los 19 municipios encontrados con fuentes termales. Se clasifica este recurso desde 2 variables: fuentes termales que presentan una alta concentración de sulfato, halladas en 6 municipios, y termales con otra composición mineral, en 13 municipios.

De esta manera, a partir de la categorización mencionada, se identificaron las termales con posibilidades de desarrollo dentro de la región de Cundinamarca.

\section{Georreferenciación de las terma- les con alternativas de desarrollo para el Turismo de Bienestar}

El trabajo de campo realizado, permitió la identificación y ubicación de 27 fuentes termales, presentes en 19 municipios del departamento de Cundinamarca; sus coordenadas se referenciaron desde las salidas de campo, por medio de un GPS. Las categorías que se han venido desarrollando, fueron la base para definir los resultados que se muestran en el mapa, de acuerdo con las siguientes convenciones:

El color morado y la imagen de color azul son los municipios que tie- nen termales con otra composición mineral.

El color amarillo y la imagen de color rojo son aquellos municipios que poseen termales con composición mineral sulfatada. Los municipios con color fucsia son aquellos que poseen las dos características mencionadas anteriormente.

En este sentido, se numera las fuentes termales del 1 a 27; se indica el nombre del recurso, el municipio donde se encuentra y la georreferenciación que indica, y su punto exacto de ubicación; a continuación, se muestra la tabla de convenciones del mapa.

El siguiente mapa es el producto de la investigación, de esta forma, se convierte en una herramienta de consulta para los actores que intervienen en el turismo, puesto que a través de esta se puede conocer los recursos existentes en Cundinamarca, con el fin de consolidar el Turismo de Bienestar; de igual forma, es el insumo que permitirá el desarrollo de propuestas e investigaciones conducentes a fortalecer este nuevo sector productivo en un futuro. 
Tabla 4. Presentación de la georreferenciación de las termales presentes en Cundinamarca.

\begin{tabular}{|c|c|c|c|}
\hline \multicolumn{4}{|c|}{ CONVENCIONES } \\
\hline 巡 & \multicolumn{3}{|l|}{ Termales con otra composición mineral } \\
\hline 巡 & \multicolumn{3}{|l|}{ Termales con composición mineral sulfatada } \\
\hline & \multicolumn{3}{|c|}{ Termales Sulfatadas y con otra composición mineral } \\
\hline PUNTO & DESCRIPCIÓN & MUNICIPIO & UBICACIÓN \\
\hline 1 & Duchas de Agua Azufrada, Hotel Abacoa & Útica & POINT(-74.480144 5.187114) \\
\hline 2 & Baños Medicinales Santa Lucía & Anapoima & POINT(-74.530748 4.553417) \\
\hline 3 & Baños Medicinales Santa Ana & Anapoima & POINT(-74.535777 4.545142) \\
\hline 4 & El Gran Pozo Azufrado & Tocaima & POINT(-74.648607 4.480854) \\
\hline 5 & Pozo Azufrado & Tocaima & POINT(-74.646756 4.482540) \\
\hline 6 & Pocitos Azufrados de Tocaima & Tocaima & POINT(-74.646380 4.478341) \\
\hline 7 & Los Chorros & Agua de Dios & POINT(-74.667209 4.363979) \\
\hline 8 & Bosques de Babilonia & Ricaurte & POINT(-74.697198 4.264224) \\
\hline 9 & Casa Blanca & Ricaurte & POINT(-74.697198 4.264224) \\
\hline 10 & Fuente Termal La Azufrada & Pandi & POINT(-74.499354 4.186657) \\
\hline 11 & Termales Rocas de Agua Caliente & Suesca & POINT(-73.720182 5.220939) \\
\hline 12 & Termales Hacienda Nápoles & Chocontá & POINT(-73.646948 5.096901) \\
\hline 13 & Termales Los Volcanes & Chocontá & POINT(-73.641658 5.092064) \\
\hline 14 & Paraíso Termal & Tibiritá & POINT(-73.526407 5.060678) \\
\hline 15 & Complejo Termal Nemocón & Nemocón & POINT(-73.876118 5.065521) \\
\hline 16 & Termales El Zipa & Tabio & POINT(-74.105169 4.923344) \\
\hline 17 & Aguas Calientes & San Francisco & POINT(-74.283828 4.934113) \\
\hline 18 & Aguas Calientes & El Rosal & POINT(-74.267391 4.891985) \\
\hline 19 & Termales Santa Bárbara & Guasca & POINT(-73.857851 4.876067) \\
\hline 20 & La Rivera & Junín / Gachetá & POINT(-73.679226 4.821092) \\
\hline 21 & Termal Las Gaviotas & Junín & POINT(-73.678293 4.822063) \\
\hline 22 & Piscina La Peña & Junín & POINT(-73.673672 4.823936) \\
\hline 23 & Termal Campo Alegre & Junín & POINT(-73.672299 4.824122) \\
\hline 24 & Termales Santa Mónica & Choachí & POINT(-73.916921 4.547729) \\
\hline 25 & Aguas Calientes & Chipaque & POINT(-74.084835 4.348872) \\
\hline 26 & Fuente Termal Las Lajas & Pandi & POINT(-74.497465 4.188833) \\
\hline 27 & Reserva Natural Aguas Calientes & Paratebueno & POINT(-73.120500 4.462222) \\
\hline
\end{tabular}

Fuente: Elaboración propia. 


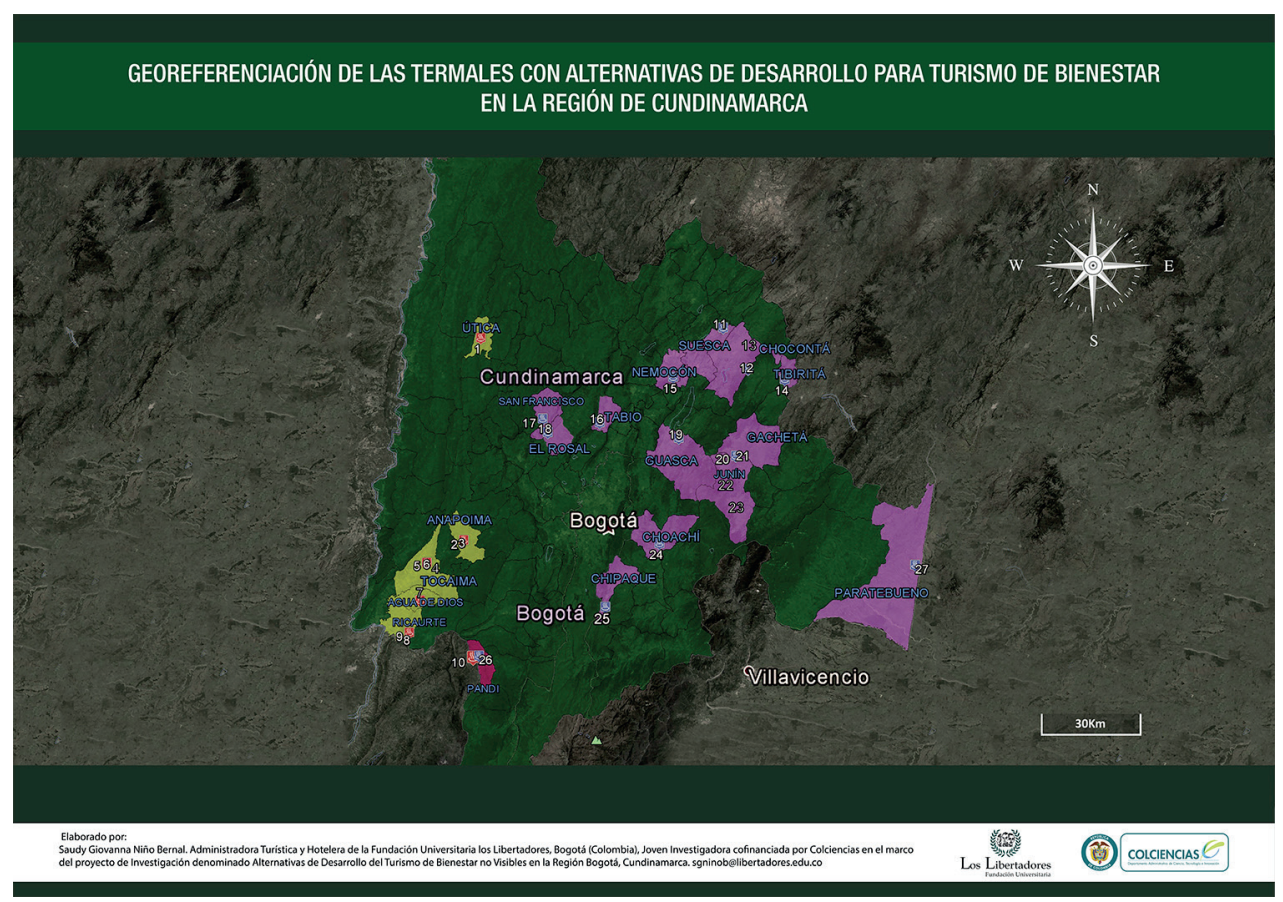

Figura 9. Georreferenciación de las termales con alternativas de desarrollo para Turismo de Bienestar en la región Cundinamarca.

Fuente: Elaboración propia.

\section{Conclusiones}

La investigación permite categorizar las termales de la región Cundinamarca, mediante el análisis de variables para identificar este recurso como una alternativa de desarrollo para el Turismo de Bienestar; es así como se georreferencian 27 termales, distribuidas en 19 municipios, que pueden ser potencializadas para el desarrollo de productos en torno de esta tipología turística.
En coherencia con lo anterior, se lleva a cabo un reconocimiento de los recursos y elementos en 39 municipios del Departamento, y se evidencia que este territorio presenta una variedad de fauna, flora, paisajes, clima, cultura, hidrología y gastronomía que puede ser desarrollada como apuesta productiva para fomentar e incrementar la llegada de turistas a este destino.

El punto central de la investigación, se enfocó en reconocer uno 
de los productos centrales para el Turismo de Bienestar: el termalismo. Se generó, como resultado, la identificación de otras alternativas que se puede desarrollar a través de este recurso. Es por esto que se propone utilizar los productos locales en el uso de terapias, masajes, mascarillas y otras actividades enfocadas hacia el bienestar.

Es así como la posibilidad de competencia de las 27 termales encontradas en 19 municipios de Cundinamarca depende de la atención prestada, inicialmente, desde el sector público; luego, por el sector privado, incluyendo la academia, asociaciones, prestadores de servicios y todos aquellos actores que intervienen, directa e indirectamente, en la actividad turística para desarrollar estrategias conducentes a consolidar estos destinos en Turismo de Bienestar; así mismo, se debe prestar una atención especial en la mejora de la infraestructura y los elementos que componen este territorio para ofrecer, de tal forma, un producto de calidad y de talla mundial.

La georreferenciación debe ser, para el administrador turístico y hotelero, una herramienta de apoyo en la ubicación y localización de los atractivos turísticos con posibilidades de potencialización y desarrollo.

\section{Referencias}

Alfaro-Valero, C. (2005). Geochemistry of Hot Spring at Cundinamarca Department, (Colombia). In Proceedings World Geothermal Congress. Recuperado de: http://www.geothermalenergy.org/pdf/IGAstandard/WGC/2005/0831.pdf

DNP - Departamento de Planeación Nacional. (2014). Plan Sectorial de Turismo 2014-2018 “Turismo para la construcción de la Paz". Recuperado de www.mincit.gov.co/minturismo/ descargar.php?id=71713

Euromonitor Internacional. (2012, marzo). Health and Wellness Tourism in Colombia. Recuperado de: http://www.euromonitor.com/health-and-wellness-tourism-in-colombia/ report

Farsari, Y., \& Prastacos, P. (2004). GIS applications in the planning and management of tourism. A companion to tourism. En A. A. Lew, C. M. Hall \& A. M. Williams (Edds.), A Companion to Tourism, pp. 596-608. Blackwell Publishing. Recuperado de http://onlinelibrary.wiley. com/doi/10.1002/9780470752272.fmatter/pdf 
Fontur. (2012). Plan de Desarrollo turístico de Cundinamarca. Recuperado de: http://www. mincit.gov.co/minturismo/descargar.php?id $=65678$

Gobernación de Cundinamarca. (2012). Plan de Desarrollo "Cundinamarca calidad de vida 2012-2016”. Recuperado de: http://www.cundinamarca.gov.co/wps/portal/Home/ SecretariasEntidades.gc/Secretariadeplaneacion/SecretariadeplaneacionDespliegue/ aspoliyplanprog_contenidos/csecreplanea_poliplanyprog_plandesarrdep

ICCA. (2001). Prácticas agrosostenibles para el departamento de Cundinamarca: manual introductorio. Bogotá: Arfo.

Ibáñez, A. (2012). Sistema de Información Geográfica para la Mejora de la Gestión y la Toma de Decisiones Difusa en Entornos Oleícolas (Tesis de doctoral, Universidad de Jaén). Recuperadode: http://sinbad2.ujaen.es/?q=es/work/2519-sistema-de-informaci\%C3\%B3ngeogr $\% \mathrm{C} 3 \% \mathrm{~A} 1$ fica-para-la-mejora-de-la-gesti\%C3\%B3n-y-la-toma-de-decisionesdifusa-en

Junior, E., \& Almeida, G. (2009). A Utilização do google earth no georreferenciamento e divulgação de pontos turísticos de cuiabá e chapada dos guimarães. Profiscientia, 4, 377-416.

Laing, J., \& Weiler, B. (2008). Mind, body and spirit: Health and wellness tourism in Asia. Asian tourism: Growth and change, 379-389.

López, J; Larios, C \& Campillo, L. (2008). Aplicación de un SIG para ubicar identificar las zonas de interés turístico y la infraestructura en la reserva ecológica cascadas de reforma, Balancán, Tabasco. Semana de Divulgación y Video Científico, 173-178.

Méndez, D. (2012). Adquisición y tratamiento de datos geográficos, mediante la aplicación de estándares y uso de software libre, que generen datos apropiados para la puesta en marcha de un Sistema de Información Geográfico Turístico, orientado a resolver consultas vía Web (Tesis de maestría, Universidad San Francisco de Quito). Recuperado de: http://repositorio. usfq.edu.ec/handle/23000/2310

Ocampo, J. (2004). Un futuro económico para Colombia. Colombia: CEPAL.

Tello, M. (2006). Las teorías del desarrollo económico local y la teoría y práctica del proceso de descentralización en los países en desarrollo. Perú: Pontificia Universidad Católica del Perú.

Tourism \& Leisure. (2013). Plan de Negocios para el subsector de Turismo de Bienestar en Colombia. Recuperado de: https://www.ptp.com.co/documentos/Resumen $\% 20$ Ejecutivo\%20190413.pdf

Recepción: 19 de marzo de 2015

Evaluación: 5 de mayo de 2015

Aceptación: 26 de mayo de 2015 